\title{
Acetabular Revision Surgery With Tantalum Trabecular Metal Acetabular Cup for Failed Acetabular Cage Reconstruction With Bone Allografts: a Retrospective Study With Mid- to Long- term Follow-up
}

\section{Chen-Heng Hsu}

Linkou Chang Gung Memorial Hospital

Chih-Chien Hu

Linkou Chang Gung Memorial Hospital

Chih-Hsiang Chang

Linkou Chang Gung Memorial Hospital

Yu-Han Chang

Linkou Chang Gung Memorial Hospital

Hsin-Nung Shih

Linkou Chang Gung Memorial Hospital

Chun-Chieh Chen ( $\nabla$ m7016@cgmh.org.tw)

Linkou Chang Gung Memorial Hospital

\section{Research Article}

Keywords: tantalum trabecular metal acetabular cup, Failed cage reconstruction, revision hip arthroplasty

Posted Date: February 2nd, 2022

DOI: https://doi.org/10.21203/rs.3.rs-1268866/v1

License: (c) (i) This work is licensed under a Creative Commons Attribution 4.0 International License.

Read Full License 


\section{Abstract}

\section{Background}

The acetabular cage reconstruction with bone allografts is one of the successful strategies to deal with the massive acetabular bone loss. However, the nonbiological fixation nature of cages can compromise long-term success. The tantalum trabecular metal acetabular cup (TM cup) has been used in acetabular revision surgery because of its increased initial stability and good bone ingrowth features. This study was to determine if the bone stock of acetabulum is enough to support the hemispheric TM cup after failed cage reconstruction with bone allografts.

\section{Methods}

We retrospectively reviewed our patients who received acetabular revision surgery with TM cups after failed cages reconstruction with bone allografts from 2006 to 2017. There were twelve patients ( 5 males and 7 females) including in this study at the mean age of 61.5 years (38 to 81 ) at re-revision surgery. The mean follow-up after re-revision surgery was 8.6 years (2.6 to 13.3). The endpoint was defined as the aseptic loosening of TM cup and reoperation for any causes. The change of bone stock of acetabulum between index revision and re-revision was assessed according to Gross classification for acetabular bone loss.

\section{Results}

One patient died after eight years of follow-up with the cause not related to hip surgery. Two patients received two-stage revision arthroplasty due to PJI after 3.2- and 9.4-years follow-up. The bone stock of acetabulum was significantly improving between index revision and re-revision surgery $(p<0.0001)$. The Kaplan-Meier survivorship was $100 \%$ when aseptic loosening as the endpoint, and $90 \%$ and $75 \%$ at fiveand ten-year when reoperation for any causes as the endpoint.

Even the cage reconstruction with bone allografts will fail eventually, the bone stock of acetabulum will improve after union and incorporation between host bone and allografts. The restored bone stocks will facilitate further revision surgery with hemispheric TM cup. The biological fixation between host bone tantalum trabecular metal will provide longstanding stability of the TM cup.

\section{Conclusions}

The results of our study offered a viable option for patients with failed cage reconstruction with bone allografts.

\section{Background}

Total hip arthroplasty (THA) is one of the most successful orthopedic interventions of its generation, but because of its popularity, an increasing number of patients undergoing total hip arthroplasty require 
revision surgery. The main reasons for implant failure in $71 \%$ of cases are aseptic loosening and associated osteolysis. ${ }^{1}$ Acetabular bone loss after THA can be a serious problem in revision surgery as bone deficiencies may limit reconstructive options, increase difficulty of operation, and necessitate autogenous or allogenic bone grafting.

Treatment options for different types of acetabular bone defects have been well-established. Most patients can have reconstruction with uncemented cups with screws with or without morselized bone grafts. Severe acetabular bone defects might require structural grafts, a cup with or without augments, a cage, or a cup-cage, depending on the type of bone defect.

Cage reconstruction is used in massive bone defects in which an uncemented porous cup cannot gain stable fixation or biologic fixation is unlikely, and in cases of pelvic discontinuity. ${ }^{2}$ However, cages have high failure rates in mid- to long-term follow-up due to a lack of biologic fixation. ${ }^{3,4}$ Although the cages themselves will fail eventually, the allografts will be protected by the cages, and thus grafts will restore bone stock of the acetabulum. ${ }^{4-6}$ We aimed to determine if after the cage is loosened, if the acetabulum bone stock is sufficient to support hemispheric cups and achieve longstanding biological fixation.

The Trabecular Metal (TM, Zimmer Biomet, Warsaw, Indiana) is made of highly porous tantalum and facilitates bone ingrowth and initial stability because of its low modulus of elasticity and high frictional characteristics. ${ }^{7-9}$ The TM cups have been demonstrated to lead to successful clinical outcomes and survivorship in revision hip surgery. ${ }^{10}$ In our previous study, we used structural allografts with the TM cup to treat Paprosky type III defects in revision THA with good clinical outcomes. ${ }^{11}$

Because of the bioactive nature and bone ingrowth properties of the $\mathrm{TM}^{10}{ }^{10}$ we suspect that $\mathrm{TM}$ cups are suitable for patients who need acetabular revision surgery due to loose cages. The purpose of this study was to report the mid- to long-term results of using trabecular metal-coated hemispherical cups (TM cup) to salvage failed cages in acetabular revision surgery.

\section{Methods}

We retrospectively reviewed the rTHA register database of our institution, and identified 2831 patients receiving rTHA in our institution from 2006 to 2017. We included patients who received acetabular revision surgery with the TM cups due to aseptic loosening of cages. The first revision surgery using cage reconstruction with bone allografts was termed "index revision," and the following revision surgery using the TM cup due to aseptic loosening of the cage was termed "re-revision."

\section{Patients}

Twelve of these patients (twelve hips), including five males and seven females, were included in this study. The mean age of the patients at index revision was $56.1 \pm 14.7$ years and $61.5 \pm 14.6$ years at rerevision. Patient demographics are summarized in Table 1. 
The acetabular bone defects at the time of index revision and re-revision were classified based on preoperative radiographs and intraoperative findings according to the Paprosky classification ${ }^{12}$ and the Gross classification for acetabular bone loss. ${ }^{13}$ Briefly, Paprosky type I defects are classified by minimal bone loss, intact dome, presented posterior wall and teardrop, and uninvolved medial wall. Type II defects are classified by distorted acetabular hemisphere, destructed dome and/or medial wall, and preserved anterior and posterior columns. Type III defects are classified by severe bone loss, destroyed acetabular rim and supporting structures. ${ }^{12}$ The Gross classification was defined as type I: no significant loss of bone stock; type II: contained loss of bone stock (cavitary); type III: uncontained loss of bone stock involving less than $50 \%$ of the acetabulum (posterior column \pm anterior column); type IV: Uncontained loss of bone stock involving more than $50 \%$ of the acetabulum (segmental loss affecting both columns); and type V: pelvic discontinuity with uncontained loss of bone stock. ${ }^{13}$

\section{Surgical procedure}

In all patients, an anterolateral approach in lateral decubitus position was chosen in index revision and rerevision surgery. Bone allografts were obtained from the bone bank of our institution. The bone bank protocol, which consisted of donor selection, harvesting, and processing of allograft followed the recommendations of the Musculoskeletal Council of the American Association of Tissue Banks. During index revision surgery, the bone defects of the acetabulum were filled with structural and morselized fresh frozen bone allografts after removal of the previous implant, debridement, and reaming to bleeding host bone. When the reasonable hip center was restored by bone grafting, the cage was placed within the acetabulum and affixed by screws. After the cage was secured, a PE liner was fixed to the cage in the appropriate orientation using bone cement. In re-revision surgery, after removal of the failed cage and debridement, the acetabulum was reamed to bleeding host bone (Figure 1A). The acetabular bone defects were assessed and the structural and/or morselized bone grafts were applied to the segmental or cavity bone defects. Then, a TM cup was press-fitted to the acetabulum.

\section{Outcome assessment}

The Gross classification was used to quantify the restoration of bone stock between index revision and re-revision surgery. The modified Harris hip score ${ }^{14}$ was used to assess clinical status. All patients were followed routinely ( 6 weeks, 3 months, 6 months, 1 year post-op, and annually thereafter). We retrospectively reviewed medical records and radiographs, including anteroposterior (AP) pelvis view and lateral hip view. Osseointegration of the TM cups after re-revision surgery was assessed according to the classification by Moore et al., ${ }^{15}$ which includes five radiographic signs: (1) absence of radiolucent lines; (2) presence of a superolateral buttress; (3) medial stress-shielding; (4) radial trabeculae; and (5) an inferomedial buttress. Loosening of TM cups was defined as migration $>3 \mathrm{~mm}$ in horizontal or vertical, change in the angle of inclination $>5^{\circ}$, and less than 2 signs of osteointegration. ${ }^{15}$ 


\section{Statistical analysis}

All statistical analyses were performed with MedCalc Statistical Software version 19.2.5 (MedCalc Software Ltd, Ostend, Belgium; https://www.medcalc.org; 2020). Kaplan-Meier survival analysis was used to estimate the survivorship of the TM cup in re-revision surgery by two endpoints: (1) aseptic loosening and (2) reoperation for any cause. Restoration of acetabular bone loss was assessed using Student's paired t-test. A p-value of $<0.05$ was considered statistically significant.

\section{Results}

One patient died after eight years of follow-up for reasons unrelated to hip surgery, but the TM cup was stable at her last follow-up. Two patients suffered from hematogenous periprosthetic joint infection (PJI) 3.2 years and 9.4 years following re-revision surgery. These two patients were received two-stage revision arthroplasty for PJI at that time. The TM cups were stable during first stage surgery for PJI. The mean interval of index revision and re-revision of these twelve patients was $5.4 \pm 3.4$ years. At the time of index revision, there were five cases of Paprosky type IIIA defects, six type IIIB defects, and one pelvic discontinuity. At re-revision, there was one Paprosky type I, four type IIA, four type IIB, two type IIC, and one type IIIA defect. Overall, bone defects were downgraded in re-revision surgery. Bone defects at index revision and re-revision surgery, according to the Gross classification are shown in Table 2. The bone stock of the acetabulum was significant restoration at the time of re-revision surgery $(p<0.001)$ according to the Gross classification (Figure 2). The mean follow-up time after re-revision surgery was 8.6 \pm yyears (Figure 3). With aseptic loosening defined as an endpoint, the Kaplan-Meier survivorship was $100 \%$. When reoperation for any cause was set as the endpoint, the Kaplan-Meier survivorship at fiveand ten-years was $90 \%$ and $75 \%$, respectively (Figure 4). There were no complications such as dislocations, sciatic nerve palsy, or surgical site infection after index revision or re-revision surgery. The mean modified Harries hip score was $85.6 \pm 4.1$ at the latest follow-up after re-revision surgery.

\section{Discussion}

Acetabular revision surgery with extensive acetabular bone defects is challenging and technically demanding. Massive bony deficiencies can arise from osteolysis after polyethylene wear, destroying the bone by loosening of an acetabular component, repeated revision of the acetabulum, or extended debridement for PJI. When dealing with massive acetabular bone loss, such as Paprosky type III acetabular defect, structural allografts not only restore bone stock, but also support new acetabular components. ${ }^{16}$ Garbuz et al. reported a high failure rate of acetabular revisions if structural allografts supported $>50 \%$ of the cup at a mean follow-up of 7 years, and more than half of failed cases showed both prosthesis and allograft failure. ${ }^{5} \mathrm{~A}$ number of previous studies have reported that reconstruction of massive acetabular bone defects using the cage with allografts could provide good initial stability. ${ }^{17,18}$ However, the inability of biologic fixation at the cage-bone interface can compromise the chances of longterm success. ${ }^{3,4}$ The cage will fail eventually because of its nonbiological fixation. 
Allogenic bone grafts are biocompatible scaffolds. Revascularization, resorption, and appositional bone growth will occur slowly. ${ }^{16}$ During acetabular revision surgery, the cage can protect the bone grafts, and the bone stock will restore over time through the union and incorporation between host bone and bone grafts. ${ }^{3}$ In our study, bone base bleeding was noted after removal of the loosened cages and reaming of the acetabulum in re-revision surgery. It indicated the host bone had incorporated with the allografts, which was implanted during index revision surgery. This bleeding living bone, remodeling from allografts, thus renovated the bone stock of the acetabulum.

The new bone stock can downgrade the bone defects of the acetabulum and provide the capableness of facilitating the re-revisions. Abolghasemian et al. reported that $48 \%$ of patients with Gross type III or higher acetabular bone defect at the time of index revision were downgraded to Gross type I or II defect at re-revision; ${ }^{19}$ moreover, $60 \%$ of hips could use hemispherical cups in re-revision surgery. In our study, all acetabular bone defects were restored through re-revision surgery, according to the Gross classification. The reconstitution of acetabulum bone stock provided an opportunity to use cementless hemispherical components in re-revision surgery.

As shown in figure 1B, although the allografts had united with the host bone, it was not all revascularized (no bleeding area, marked as *), and allograft had been absorbed. A revascularized allograft predisposes weakening and loosening of the structure. ${ }^{20}$ Although there was Gross type I or II acetabular bone defects in re-revision surgery, the bleeding host bone may not be strong enough to support a conventional uncemented cup. ${ }^{10}$

The TM is made of tantalum, a highly porous biomaterial (porosity $70 \%-80 \%$ ), in $3 \mathrm{D}$ architecture. One study using an animal model reported bone ingrowth within two weeks, and substantial ingrowth occurred within four weeks. ${ }^{7}$ Other studies have report that the TM cup is suitable for acetabular revision surgery with massive bone loss, even when there is less than $50 \%$ of acetabulum host bone. ${ }^{10,11,21}$ Kosashvili et al. reported 15 patients receiving non-buttressed TM cup reconstruction for failed acetabular cages with a mean follow-up time of 48.3 months (range, 24-72 months). Of these 15 patients, 12 did not have cup loosening at the end of the follow-up period. ${ }^{22}$ In the current study, we observed no radiological failures due to aseptic loosening after re-revision surgery at a mean follow-up time of 8.7 years follow-up, and only two hips failed due to infection after 3.2 and 9.4 years follow-up. The TM cup provides higher mechanical stability and a more favorable environment for bone incorporation with the metal cup. ${ }^{23,24}$ For these reasons, we use the TM cup in re-revision surgery after the failed cage reconstruction with bone allografts in our clinical practice with good results.

There are some limitations to our study which need to be addressed. First, the number of patients this study was relatively small, despite the high-volume of referrals to our institution. Also, we were not able to compare this technique to other revision techniques as this was a retrospective study, and this was not a randomized study thus there may have been selection bias. We used TM cups to salvage failed cages based on evaluation of pre-operative radiographs and assessing acetabular bone defects and the quality 
of bleeding living bone intra-operation. However, taken together, our study suggests that TM cup reconstruction is a viable option for re-revision surgery after failed cage reconstruction with bone allografts.

\section{Conclusions}

This study suggests that using cages combining with allografts in acetabular revision surgery with massive bone loss can restore acetabular bone stock. Although the cages will eventually fail, the reconstituted bone stock can help with future revisions. The TM cup is a reliable option and relatively easier procedure for re-revision surgery after the failed cage reconstruction with bone allografts. The benefit of TM cups for biological fixation can provide a long-lasting construction after re-revision surgery.

\section{List Of Abbreviations}

Tantalum trabecular metal acetabular cup (TM cup), Total hip arthroplasty (THA)

\section{Declarations}

\section{-Ethics approval and consent to participate:}

The ethics committee of the Chang Gung Memorial Hospital (CGMH), Kweishian, Taoyuan, Taiwan approved the study design on Sep 9th, 2016 (CGMH IRB No: 201601034B0) and waived the requirement for obtaining informed consent and patient records.

\section{-Consent for publication:}

The authors confirmed that all methods were carried out in accordance with relevant guidelines and regulations. Consent for publication is not applicable to our study

\section{-Availability of data and materials:}

The authors confirm that the data supporting the findings of this study are available within its supplementary file.

\section{-Competing interests:}

The authors declare that they have no known competing financial interests or personal relationships that could have appeared to influence the work reported in this paper.

\section{-Funding:}

This research received no specific grant from any funding agency in the public, commercial, or not-forprofit sectors. 


\section{-Authors' contributions:}

Hsin-Nung Shih conceived of the presented idea. Chen-Heng Hsu, Chun-Chieh Chen developed the theory and performed the computations. Chun-Chieh Chen verified the analytical methods and supervised the findings of this work. All authors discussed the results and contributed to the final manuscript.

\section{-Acknowledgements:}

The authors thank the Bone and Joint Research Center, Chang Gung Memorial Hospital for the clinical data support and Enago (www.enago.tw) for their English language review.

\section{-Consent for Publication:}

The authors declared no potential conflicts of interest with respect to the research, authorship, and/or publication of this article.

\section{References}

1. Herberts $P$, Malchau H. Long-term registration has improved the quality of hip replacement: a review of the Swedish THR Register comparing 160,000 cases. Acta Orthop Scand 2000;71:111-21.

2. Berry DJ. Antiprotrusio cages for acetabular revision. Clin Orthop Relat Res 2004;420:106-12.

3. Goodman S, Saastamoinen H, Shasha N, Gross A. Complications of ilioischial reconstruction rings in revision total hip arthroplasty. J Arthroplasty 2004;19:436-46.

4. Saleh KJ, Jaroszynski G, Woodgate I, Saleh L, Gross AE. Revision total hip arthroplasty with the use of structural acetabular allograft and reconstruction ring: a case series with a 10-year average followup. J Arthroplasty 2000;15:951-8.

5. Garbuz D, Morsi E, Gross AE. Revision of the acetabular component of a total hip arthroplasty with a massive structural allograft: Study with a minimum five-year follow-up. J Bone Joint Surg Am 1996;78:693-7.

6. Perka C, Ludwig R. Reconstruction of segmental defects during revision procedures of the acetabulum with the Burch-Schneider anti-protrusio cage. J Arthroplasty 2001;16:568-74.

7. Bobyn JD, Stackpool GJ, Hacking SA, Tanzer M, Krygier JJ. Characteristics of bone ingrowth and interface mechanics of a new porous tantalum biomaterial. J Bone Joint Surg Br 1999;81:907-14.

8. Garcia-Cimbrelo E. Porous-coated cementless acetabular cups in revision surgery: a 6- to 11-year follow-up study. J Arthroplasty 1999;14:397-406.

9. Unger AS, Lewis RJ, Gruen T. Evaluation of a porous tantalum uncemented acetabular cup in revision total hip arthroplasty: clinical and radiological results of 60 hips. J Arthroplasty 2005;20:1002-9.

10. Lakstein D, Backstein D, Safir O, Kosashvili Y, Gross AE. Trabecular Metal cups for acetabular defects with $50 \%$ or less host bone contact. Clin Orthop Relat Res 2009;467:2318-24. 
11. Chang $\mathrm{CH}$, Hu CC, Chen CC, Mahajan J, Chang Y, Shih HN et al. Revision total hip arthroplasty for Paprosky Type III acetabular defect with structural allograft and tantalum trabecular metal acetabular cup. Orthopedics 2018;41:e861-7.

12. Paprosky WG, Perona PG, Lawrence JM. Acetabular defect classification and surgical reconstruction in revision arthroplasty. A 6-year follow-up evaluation. J Arthroplasty 1994;9:33-44.

13. Saleh KJ, Holtzman J, Gafni ASaleh L, Jaroszynski G, Wong P, Woodgate I et al. Development, test reliability and validation of a classification for revision hip arthroplasty. J Orthop Res 2001;19:50-6.

14. Dawson J, Fitzpatrick R, Carr A, Murray D. Questionnaire on the perceptions of patients about total hip replacement. J Bone Joint Surg Br 1996;78:185-90.

15. Moore MS, McAuley JP, Young AM, Engh CA Sr. Radiographic signs of osseointegration in porouscoated acetabular components. Clin Orthop Relat Res 2006;444:176-183.

16. Pierannunzii L, Zagra L. Bone grafts, bone graft extenders, substitutes and enhancers for acetabular reconstruction in revision total hip arthroplasty. EFORT Open Rev 2017;1:431-9.

17. Gross AE, Goodman S. The current role of structural grafts and cages in revision arthroplasty of the hip. Clin Orthop Relat Res 2004;429:193-200.

18. Hosny HAH, El-Bakoury A, Fekry H, Keenan J. Mid-term results of graft augmentation prosthesis ii cage and impacted allograft bone in revision hip arthroplasty. J Arthroplasty 2018;33:1487-93.

19. Abolghasemian M, Sadeghi Naini M, Tangsataporn S, Lee P, Backstein D, Safir $O$ et al. Reconstruction of massive uncontained acetabular defects using allograft with cage or ring reinforcement: an assessment of the graft's ability to restore bone stock and its impact on the outcome of re-revision. Bone Joint J 2014;96-B:319-24.

20. Enneking WF, Burchardt H, Puhl JJ, Piotrowski G. Physical and biological aspects of repair in dog cortical-bone transplants. J Bone Joint Surg Am 1975;57:237-52.

21. Clement RG, Ray AG, MacDonald DJ, Wade FA, Burnett R, Moran M. Trabecular metal use in Paprosky Type 2 and 3 acetabular defects: 5-year follow-up. J Arthroplasty 2016;31:863-7.

22. Kosashvili Y, Safir O, Backstein D, Lakstein D, Gross AE. Salvage of failed acetabular cages by nonbuttressed trabecular metal cups. Clin Orthop Relat Res 2010;468:466-71.

23. Christie MJ. Clinical applications of trabecular metal. Am J Orthop (Belle Mead NJ) 2002;31:219-20.

24. Meneghini RM, Meyer C, Buckley CA, Hanssen AD, Lewallen DG. Mechanical stability of novel highly porous metal acetabular components in revision total hip arthroplasty. J Arthroplasty 2010;25:33741.

\section{Tables}

Table 1. Patient demographics 


\begin{tabular}{|ll|}
\hline Variable & All hips (n=12) \\
\hline Sex, $\mathbf{n}$ & \\
\hline Male & 5 \\
\hline Female & 7 \\
\hline Mean age at index revision surgery, years (SD) & 56.1 (14.7) \\
\hline Mean age at re-revision surgery, years (SD) & $61.5(14.6)$ \\
\hline Mean interval between index revision and re-revision, years (SD) & $5.4(3.4)$ \\
\hline Mean follow-up after re-revision, years (SD) & $8.6(4)$ \\
\hline Reason for index revision surgery, $\mathbf{n}$ & \\
\hline Loose acetabular component & 9 \\
\hline Second-stage re-implantation for infection & 3 \\
\hline Paprosky classification for index revision, $\mathbf{n}$ & \\
\hline IIIA & 5 \\
\hline IIIB & 6 \\
\hline Pelvic discontinuity & 1 \\
\hline Paprosky classification for re-revision, $\mathbf{n}$ & 2 \\
\hline I & 4 \\
\hline IIA & 4 \\
\hline IIB & 2 \\
\hline IIC & 4 \\
\hline IIIA & \\
\hline
\end{tabular}

Table 2. Gross classification of acetabular bone loss at index and re-revision surgery

\begin{tabular}{|c|c|c|c|c|c|}
\hline & Defect & & & & \\
\hline Surgery & Type I & Type II & Type III & Type IV & Type V \\
\hline Index revision & & & 1 & 10 & 1 \\
\hline Re-revision & 4 & 3 & 5 & & \\
\hline
\end{tabular}




\section{Figures}

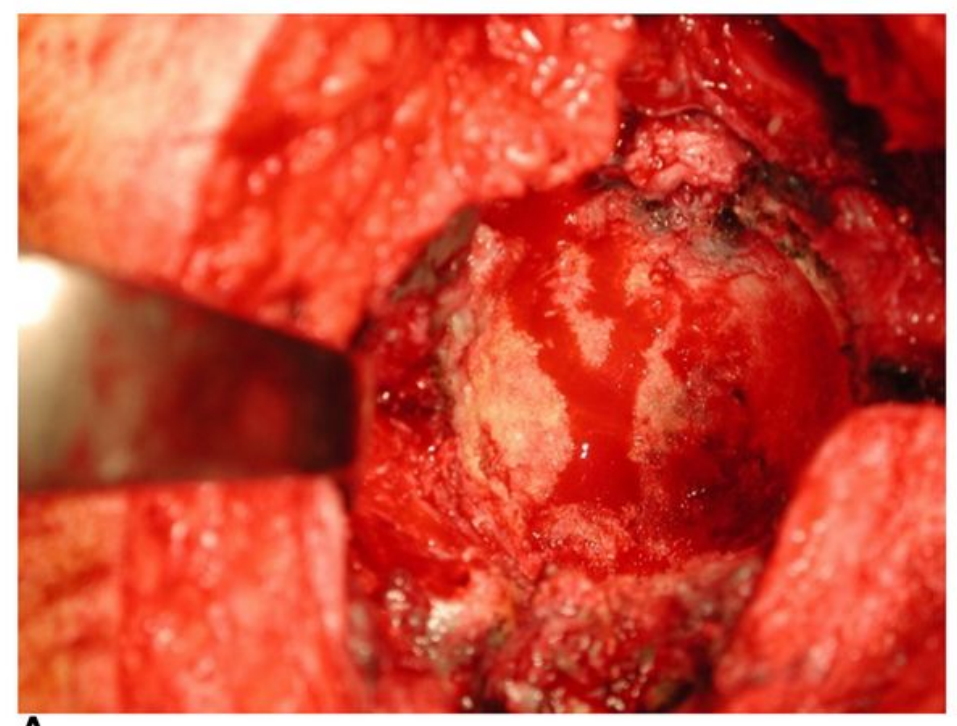

A

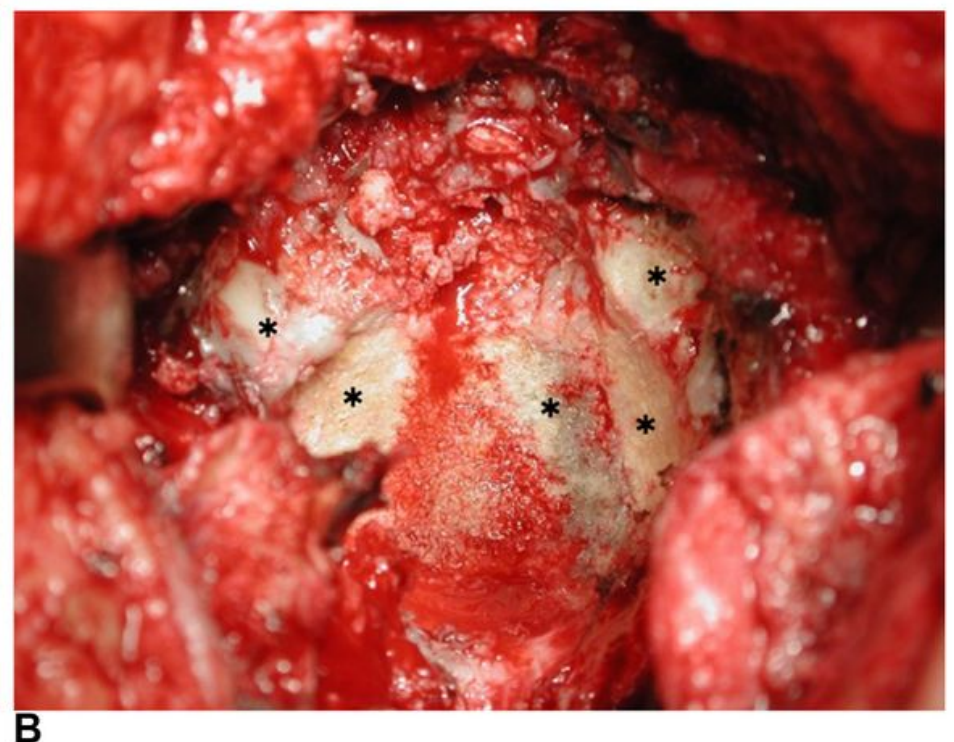

Figure 1

A) The host bone of acetabulum was bleeding after reaming in re-revision surgery, indicating revascularization of allografts. B) The allografts, which were implanted in index revision, had nonvascularized areas (marked with *) during re-revision surgery. 


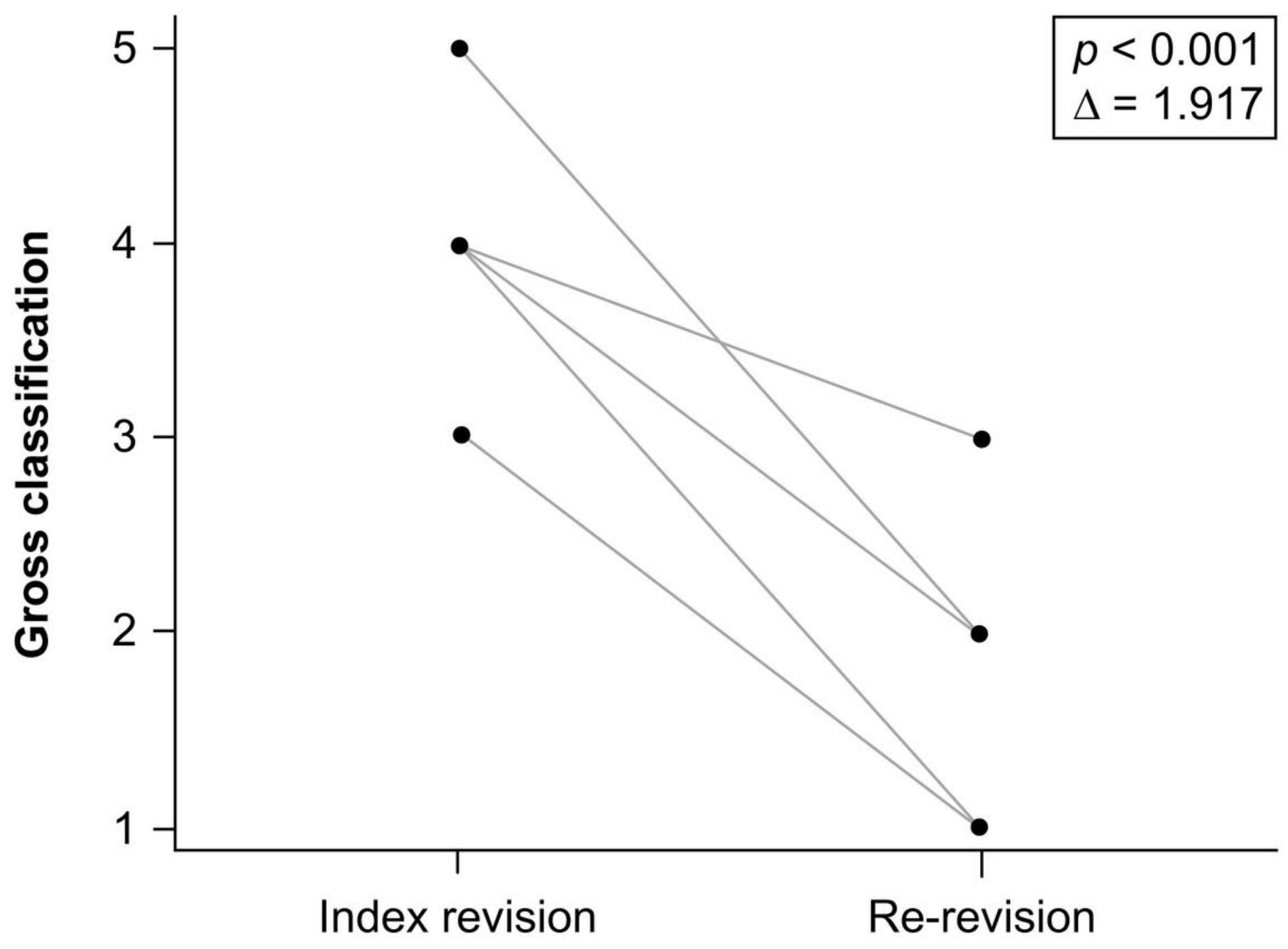

Figure 2

Acetabulum bone loss significantly decreased between index revision and re-revision according to Gross classification $(p<0.001)$. 

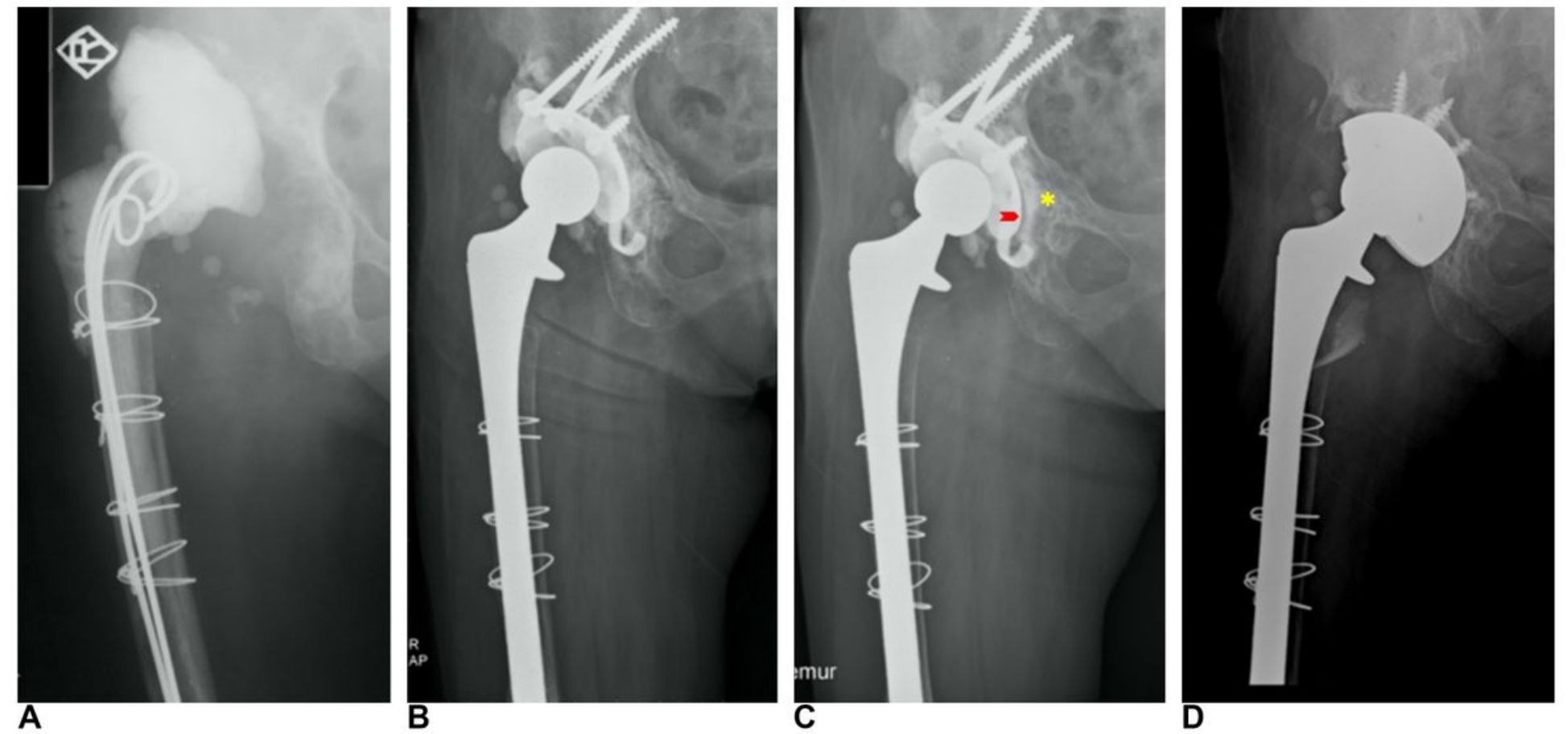

\section{Figure 3}

A) Paprosky type IIIA acetabular bone defect after operation for periprosthetic joint infection. B) Reconstruction with the cage and bone allografts. C) The cage failed eight years after index revision. There was radiolucent line between the cage and host bone (arrow), and union of the host bone and allografts (*). D) The TM cup remained stable after nine years of follow-up after re-revision surgery. 


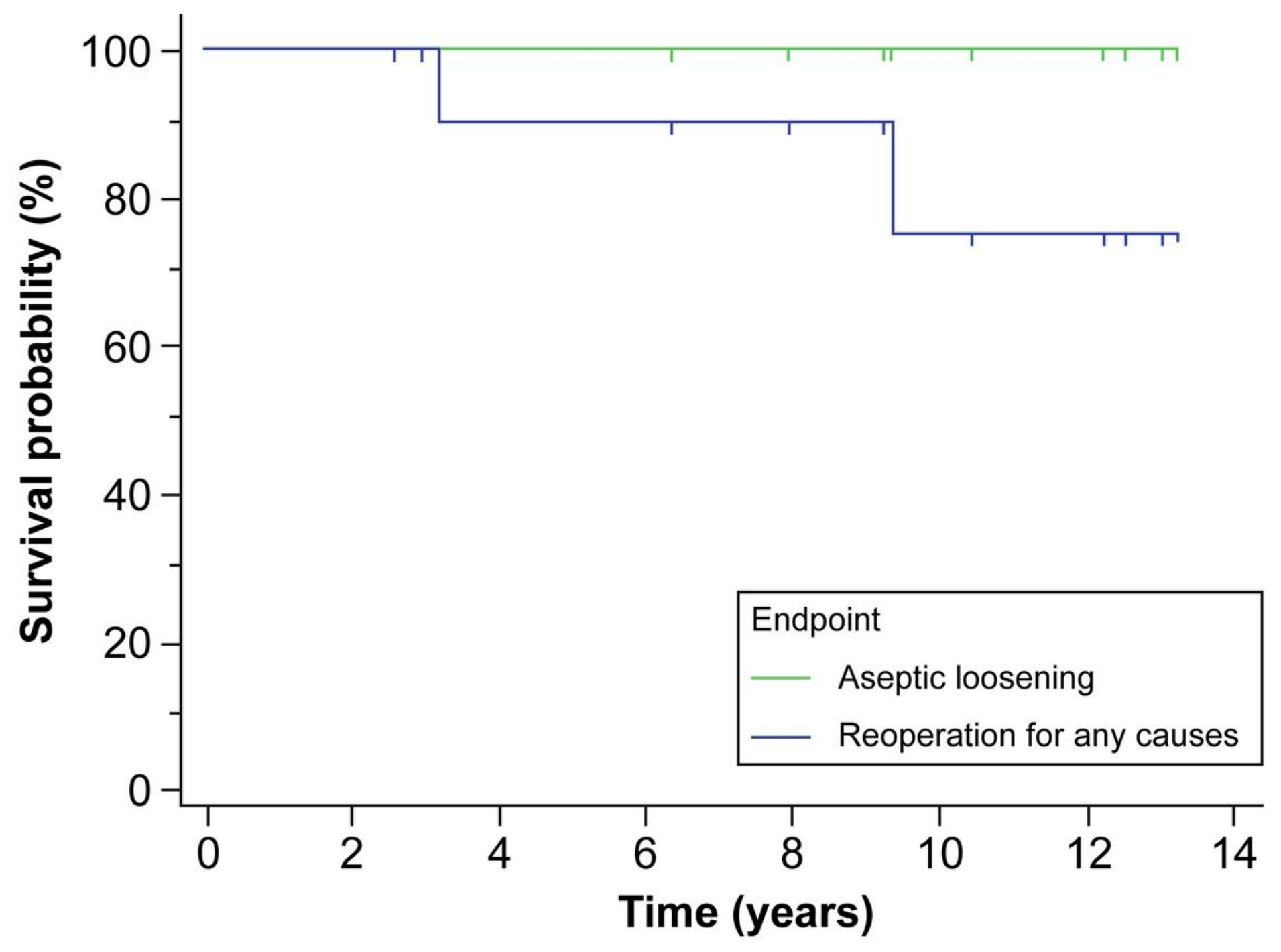

Figure 4

The Kaplan-Meier survivorship was $100 \%$ with aseptic loosening as the endpoint, and $90 \%$ and $75 \%$ at five- and ten-years respectively with the reoperation for any causes as the endpoint.

\section{Supplementary Files}

This is a list of supplementary files associated with this preprint. Click to download.

- RawData.xlsx 